\title{
The Effect of Sound Reproduction Method on Performance in Sound Source Localization by Visually Impaired and Normally Sighted Subjects
}

\author{
A. FurmanN*, E. Skrodzka, Ł. Giżewski and P. Nowotny \\ Institute of Acoustics, A. Mickiewicz University, Umultowska 85, 61-614 Poznań, Poland
}

\begin{abstract}
The study was undertaken to check the effect of 3D sound recording and reproduction methods on performance in localization of sound source by the visually impaired and normally sighted subjects. The performance was evaluated on the basis of the ability to identify the direction from which the sound comes and the direction of its propagation. The experiment involved a test in which the subjects were exposed to sounds reproduced by two methods of spatial reproduction: binaural (headphones) and Ambisonics (loudspeaker). The binaural recordings were reproduced through two types of headphones, open and closed ones, and the recordings were made with the help of a dummy head. The recordings for loudspeaker reproduction were made with the use of two microphone matrices, Octava and Panasonic. The subjects were adults and children, including blind and VIC. They had otologically normal hearing. The test included realization of three tasks. The first concerned identification of the direction from which sound has come from an immovable source, a rattle or a drum. In the second and third task, the subjects were asked to indicate the direction of motion of the source of sound (vehicle). For the adults, the method of recording and reproduction of sound had little effect on the test results. For the VIC, a significant difference was noted between the results of headphone and loudspeaker exposure, to the advantage of the headphone exposure. The method of binaural recording and sound exposure through headphones seems much more effective for auditory training of VIC.
\end{abstract}

DOI: 10.12693/APhysPolA.123.988

PACS: 43.66.Pn

\section{Introduction}

The ability to localize the sound source is very important both for humans and animals. It permits spatial orientation, localization of the site of the sound source, its distance and direction of its motion. The localization of the sound source is made on the basis of comparison of information reaching both ears, i.e. interaural time differences and interaural intensity differences. It is also possible to localize the sound source on the basis of the information reaching only one ear (monaural information) $[1,2]$. In literature, the term "lateralization" has also been used to describe the identification of site of the source of sound exposure through headphones in which the sound plan is usually imagined "inside" the head. In our study, although we also use the headphone sound exposure, we will use the term "localization" of sound source. Our argument is that upon presentation of binaural recordings, the sound scene is localized by the listener "outside" the head, analogously as upon sound exposure through loudspeakers or real sound exposure.

The study has been inspired by the project on development of auditory training of spatial orientation for the blind and visually impaired persons. The ability of spatial orientation is of substantial importance for the visually handicapped individuals improving their functioning

*corresponding author; e-mail: furmann@amu.edu.pl in everyday life, increasing the safety of independent mobility, and recognition of the environment. The possibility of simulation of the actual acoustic events by headphones and loudspeakers can help in training the hearing sense for this purpose. Such a training could be implemented at schools to make the process of the spatial orientation teaching faster, more effective, and safer [3-5].

Modern methods of sound recording and reproduction have been designed to create the sensation of space (3D). The methods are well theoretically described and one of the most advanced are binaural techniques for headphone exposure and variations of the Ambisonics technique for loudspeaker exposure. Literature does not provide the unambiguous and objective data on the effectiveness of binaural and Ambisonics techniques in true reproduction of sound space.

The main aim of the study was to check the effect of $3 \mathrm{D}$ sound recording and reproduction methods on performance in localization of sound source and identification of the direction of its movement by the visually impaired and normally sighted subjects. The study was performed with the use of two microphone matrices (Octava i Panasonic) while the Ambisonics method was applied and two types of headphones (open and closed) when the binaural method was used.

\subsection{Binaural method of sound recording and reproduction}

The binaural method of recording and reproduction is designed for listening through headphones. The acoustic event is recorded and then reproduced in such a way 
that the listener has an impression of being at the site of recording. From the physical point of view, the listener gets to his/her ears through the headphones a signal identical to that he/she would get if he/she was at the site of recording [6]. Considering the acoustic wave reaching the listener as an input signal to some system and the signal received at the tympanic membranes as the output signal, head and ears can be treated as a system of the self-tuning filters connected to a common input. Selftuning can be understood in such sense that the filters automatically superimpose on the signal a transfer function depending on the angle at which the wave reaches the system of head and ears. Transfer functions of the two filters are usually different. Hence, besides the monaural information provided to each ear, also binaural information in the form of interaural difference between the signals reaching each tympanic membrane is provided. Since the linear distortions introduced by the two filters are highly dependent on the direction and angle of incidence, the temporal and spectral information on the position of sound sources relative to the head-ears system is encoded in them. The idea of binaural method of sound recording is to obtain the transfer function of the two filters, superimpose them on the signal, and transfer through the headphones to the tympanic membranes of the listener. In this way, the listener gets full spatial information on the transmitted sound and has an impression of perception of natural sound. Transfer function of each of these filters is called the head-related transfer function (HRTF). In this study, the superposition of the HRTF onto the sound signal was obtained by recording the signal with the use of a head model with auricles and microphones placed at sites of tympanic membranes, the so called dummy head. The most important drawback of this method is necessity to use a dummy head characterized by the averaged HRTF. The shape of the head, auricles, and external acoustic duct are different for each individual, so the use of the averaging means that the hearing sensations are different for each listener, the spatial character can be disturbed, front-back and up-down confusions may appear as related to the cone of confusion [6]. Moreover, the method can be applied only for the sound exposure through headphones which means that the listener cannot turn the head towards the virtual sound source and hears the spatial sensation only in the way it has been recorded. However, listening through headphones is relatively cheap and is not restricted to one room with a sound installation.

\subsection{Ambisonics}

Another method of sound presentation to the listener in such a way that the signals perceived would simulate certain impressions allowing estimation of a distance of the source of sound and its direction involves the use of loudspeakers. There are some loudspeaker methods aimed to simulate spatial acoustic impressions, e. g. amplitude difference stereophony (intensity stereophony) [7], vector-base amplitude panning (VBAP) [8], 5.1-
Surround [9], synthesis with spherical harmonics (ambisonics) [10], synthesis by means of boundary method (wave-field synthesis, (WFS)) [11], and binaural-cue selection methods [12]. These methods are characterized by a parameter describing the area of the best reproduction of spatial impressions called the sweet-spot. Each of them has advantages and disadvantages [12]. In the present paper, the ambisonics method is investigated. It is one of the most popular methods of spatial sound reproduction. The microphone technique used in ambisonics and construction of the microphone matrix for sound recording have been described by many authors [12-14].

The most important merit of this method is the possibility of reproduction of the spatial signal in three dimensions $(3 \mathrm{D})$. In this study, the sound reproduction refers to the process of sound (sound event) recording and then its faithful reproduction with the help of loudspeakers with no simulation procedures. An important good point of this method is easy adaptation of the recorded signal to a given configuration of the listening system. The adaptation can be performed by analog or digital devices using the applications generally available from the internet. The main drawback of the ambisonics is a very narrow sweet-spot and the fact that the acoustic events are reproduced at a distance equal to that from the loudspeakers. The presence of the listener in the acoustic field is the source of errors related to the sound direction, which gives a spread signal, especially from lateral directions [12].

\section{Test signals, registration and reproduction, listening tests, and subjects}

The experiments were performed with the sounds generated by immovable and moving sources.

\subsection{Test signals}

When working with immovable sound sources, the goal was to ensure the reproducibility of signals. The sounds of two simple instruments, a rattle-box and a drum, were recorded. These sounds differ considerably in the spectral composition (Fig. 1). To get signal samples without the reflected sounds and background noise, the recording was made in an anechoic chamber with an omnidirectional microphone BK 4198 connected to a 5-channel interface BK 3560-B and a PC unit with the BK PULSE software. Thanks to the above means, the sound samples recorded could be reproduced through a loudspeaker mounted on a boom permitting its movement along a circle with the dummy head in its center (head and torso simulator BK 4100D) and microphone matrices.

A series of monophonic samples of the rattle-box and the drum recorded in the anechoic chamber was then reproduced through a loudspeaker placed at a certain angle with respect to the axis passing through a nose of the dummy head $\left(0^{\circ}, 45^{\circ}, 90^{\circ}, 135^{\circ}, 180^{\circ}, 225^{\circ}, 270^{\circ}, 315^{\circ}\right.$, and above the dummy head) and then recorded by the 


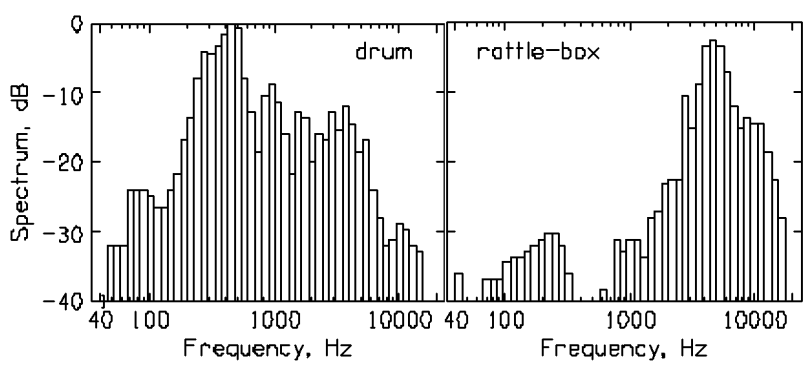

Fig. 1. Spectrum of the drum (left) and the rattle-box (right).

dummy head or microphone matrices. The sound recording and reproduction was carried out with the use of multichannel DAW Samplitude 8 software. The dummy head was connected to the computer via the BK interface, so that the PULSE system could adjust the microphone calibration automatically. The microphone matrices were connected to an 8-channel interface Focusrite Saffire PRO 40; the calibration was made by a BK 4231 calibrator.

For recordings of moving sound sources, the dummy head and the microphone matrices were places in a quiet part of the city. The sounds generated by a single car or two cars moving simultaneously at the speed of $50 \mathrm{~km} / \mathrm{h}$ were recorded. As the sound registration was made to simulate the car passing near a person standing at a pedestrian crossing or a traffic island, the recording was made for the sound source moving in two possible directions on four sides of the recording system (right $(\mathrm{R})$, left $(\mathrm{L})$, front $(\mathrm{F})$, back (B)). The same equipment was used as for recording sound events generated by immovable sources.

\subsection{Binaural method}

The sound files were recorded by the dummy head in the stereo format * ${ }^{*}$ wav, 24-bit, $44100 \mathrm{~Hz}$. Then the files were arranged into a test and a voice of a lecturer giving instructions was added. For binaural sound presentation, open headphones Sennheiser HD 600 and closed headphones Bayerdynamic DT 150 were used.

\subsection{Ambisonics}

Two microphone matrices with different transducers were used, hence they had different sizes. The first matrix was constructed by the authors of Panasonic WM55A103 electret microphones.

The transducer capsules were joined by their back rims so that their fronts made a basis of a virtual regular tetrahedron (Fig. 2a). The preamplifier containing an electronic system was constructed. It realized mathematical operations needed for Ambisonics so that at the output of the preamplifier the signal was obtained in the B-format, so in the form desired.

The second matrix (Fig. 2b) was constructed of two pairs of Octava MK-012 microphones. The microphones were held in the proper arrangement by mounting to a

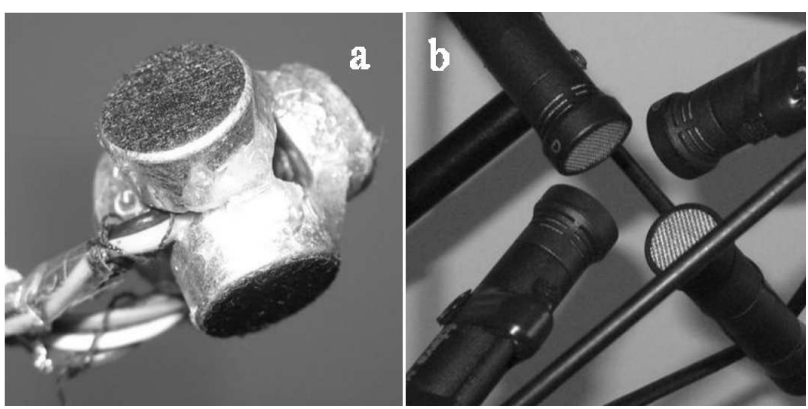

Fig. 2. Microphone matrix constructed of: (a) Panasonic devices, (b) Octava devices.

skeleton of a regular tetrahedron (constructed by the authors). To each vertex of the tetrahedron, a rod was attached along the normal and to these rods the microphones were mounted in such a way that their capsules were inside the tetrahedron and each capsule was directed towards a different basis of the solid.

The sound files were recorded in monophonic format *.wav, 24-bit, $44100 \mathrm{~Hz}$. The files from the microphone matrices were converted in the VVmic software. After setting proper filter parameters, correction parameters, type of matrix, and directivity, the software converted *.wav files to eight monophonic files, assigned to proper channels. The files were ready to be used in the test after removing unnecessary parts. The sounds prepared in the above-described way were arranged to make a test; the voice of lecturer was added. The test was prepared in the Samplitude 8 software. Outputs of the Samplitude tracks were assigned to the proper output channels of Focusrite interface to which the loudspeakers were also connected.

The sounds from the microphone matrices were reproduced by eight active QSC K-series loudspeakers arranged along a circle of $1.5 \mathrm{~m}$ diameter around the listening position. The first loudspeaker was in front of the listener and the other ones were arranged clockwise at the same distances, at the height of the listener's head.

\subsection{Listening tests and subjects}

The test was composed of samples of sounds generated by immovable and moving sources. The immovable sources were simple musical instruments, a rattle-box and a drum. The sounds produced by them were recorded in the anechoic chamber at different angles with respect to the dummy head $\left(0^{\circ}, 45^{\circ}, 90^{\circ}, 135^{\circ}, 180^{\circ}, 225^{\circ}, 270^{\circ}\right.$, $315^{\circ}$ and above the head - top). The movable sources of sound were motor vehicles moving from different directions with respect to the recording system. The listeners were given two tasks concerning identification of the directions of moving motor vehicles; in the first task, single cars moved, while in the second task, two cars moved simultaneously. When the two cars were moving, they were always on the opposite sides with the respect to the recording system and the senses of their motion vectors were the opposite. If one car was moving in front 
of the microphone from right to left, the other car was moving behind the microphone from left to right, and if one car was moving on the right of the microphone from the front to back, the other car was moving on the left of the microphone from the back to front. The listeners were informed about this prior to taking the test. All test signal or pairs of test signals were presented three times in random sequence. Prior to the test, examples of each acoustic situations were presented. The listener was asked to identify the direction of the sound or the direction of the car movement. The persons with normal eyesight were asked to mark the answer in the answer sheet, while the visually impaired subjects were asked to tell their answers to the experimenter who marked it down. The test lasted from 13 to 25 minutes, depending on the pace of work of the subjects.

The subjects were 9 adults (NSA), 9 children with normal eyesight (NSC), and 8 visually impaired children (VIC) of different degree of disability. The children were from 13 to 15 years of age. All subjects had otologically normal hearing.

\section{Results and discussion}

The effect of the signal used, the method of sound reproduction, and directions of sound presentation on the effectiveness of localization of sound source by the groups of subjects in particular tasks is illustrated by the results presented in Figs. 3-5. The bars stand for 95\% confidence intervals and points denote the arithmetic means. The effectiveness of correct localization is described by the percentage of correct answers. Table I presents results of statistical analysis for the task with immovable sources of sound, while Table II - those for the tasks with moving sound sources. Table III presents ANOVA anal-

TABLE I

Statistical significance $p$ of differences in the means of correct answers between tested groups of subjects in the test for localization of stationary sound source; nssd denotes "no statistically significant differences".

\begin{tabular}{|c|c|c|c|c|c|c|c|c|c|}
\hline \multirow[t]{2}{*}{ Groups } & \multicolumn{9}{|c|}{ Stationary sound sources } \\
\hline & \multicolumn{5}{|c|}{ Drum } & \multicolumn{4}{|c|}{ rattle-box } \\
\hline $\mathrm{VIC} / \mathrm{NSA}$ & \multicolumn{5}{|c|}{0.01} & \multicolumn{4}{|c|}{ nssd } \\
\hline $\mathrm{VIC} / \mathrm{NSC}$ & \multicolumn{5}{|c|}{0.01} & \multicolumn{4}{|c|}{0.05} \\
\hline \multirow[t]{3}{*}{$\mathrm{NSC} / \mathrm{NSA}$} & \multicolumn{5}{|c|}{0.01} & \multicolumn{4}{|c|}{0.01} \\
\hline & \multicolumn{9}{|c|}{ Method } \\
\hline & \multicolumn{3}{|c|}{ open } & \multicolumn{2}{|c|}{ close } & \multicolumn{2}{|c|}{ Octava } & \multicolumn{2}{|c|}{ Panasonic } \\
\hline $\mathrm{VIC} / \mathrm{NSA}$ & \multicolumn{3}{|c|}{0.05} & \multicolumn{2}{|c|}{0.01} & \multicolumn{2}{|c|}{ nssd } & \multicolumn{2}{|c|}{0.01} \\
\hline $\mathrm{VIC} / \mathrm{NSC}$ & \multicolumn{3}{|c|}{0.01} & \multicolumn{2}{|c|}{0.01} & \multicolumn{2}{|c|}{ nssd } & \multicolumn{2}{|c|}{ nssd } \\
\hline \multirow{3}{*}{$\mathrm{NSC} / \mathrm{NSA}$} & & 0.01 & & & 01 & & ssd & & .01 \\
\hline & \multicolumn{9}{|c|}{ Sound source angle [degrees] } \\
\hline & 0 & 45 & 90 & 135 & 180 & 225 & 270 & 315 & top \\
\hline $\mathrm{VIC} / \mathrm{NSA}$ & 0.01 & nssd & nssd & 0.01 & nssd & 0.05 & nssd & 0.01 & nssd \\
\hline $\mathrm{VIC} / \mathrm{NSC}$ & 0.01 & nssd & nssd & 0.01 & 0.01 & 0.01 & nssd & nssd & 0.01 \\
\hline $\mathrm{NSC} / \mathrm{NSA}$ & 0.01 & nssd & nssd & nssd & nssd & nssd & nssd & 0.01 & nssd \\
\hline
\end{tabular}

TABLE II

Statistical significance of differences in the means of correct answers between tested groups of subjects in the test for identification of direction of movement of one or two vehicles.

\begin{tabular}{|c|c|c|c|c|c|c|c|c|}
\hline \multirow[t]{2}{*}{ Groups } & \multicolumn{8}{|c|}{ Number of vehicles } \\
\hline & \multicolumn{4}{|c|}{ single } & \multicolumn{4}{|c|}{ two } \\
\hline $\mathrm{VIC} / \mathrm{NSA}$ & \multicolumn{4}{|c|}{ nssd } & \multicolumn{4}{|c|}{ nssd } \\
\hline $\mathrm{VIC} / \mathrm{NSC}$ & \multicolumn{4}{|c|}{ nssd } & \multicolumn{4}{|c|}{0.05} \\
\hline \multirow[t]{3}{*}{$\mathrm{NSC} / \mathrm{NSA}$} & \multicolumn{4}{|c|}{0.01} & \multicolumn{4}{|c|}{0.001} \\
\hline & \multicolumn{8}{|c|}{ Single vehicle/method } \\
\hline & \multicolumn{2}{|c|}{ Open } & \multicolumn{2}{|c|}{ Close } & \multicolumn{2}{|c|}{ Octava } & \multicolumn{2}{|c|}{ Panasonic } \\
\hline $\mathrm{VIC} / \mathrm{NSA}$ & \multicolumn{2}{|c|}{0.01} & \multicolumn{2}{|c|}{ nssd } & \multicolumn{2}{|c|}{0.01} & \multicolumn{2}{|c|}{0.001} \\
\hline $\mathrm{VIC} / \mathrm{NSC}$ & \multicolumn{2}{|c|}{0.001} & \multicolumn{2}{|c|}{0.01} & \multicolumn{2}{|c|}{ nssd } & \multicolumn{2}{|c|}{0.01} \\
\hline \multirow[t]{3}{*}{ NSC/NSA } & $\mathrm{ns}$ & sd & & sd & & 05 & & sd \\
\hline & \multicolumn{8}{|c|}{\begin{tabular}{|c|} 
Single vehicle/direction of motion \\
\end{tabular}} \\
\hline & \multicolumn{8}{|c|}{$\mathrm{R} / \mathrm{B}-\mathrm{F}|\mathrm{R} / \mathrm{F}-\mathrm{B}| \mathrm{B} / \mathrm{R}-\mathrm{L} / \mathrm{L} / \mathrm{F}-\mathrm{B}|\mathrm{L} / \mathrm{B}-\mathrm{F}| \mathrm{F} / \mathrm{L}-\mathrm{R}|\mathrm{B} / \mathrm{L}-\mathrm{R}| \mathrm{F} / \mathrm{R}-\mathrm{B}$} \\
\hline $\mathrm{VIC} / \mathrm{NSA}$ & 0.001 & nssd & nssd & nssd & nssd & nssd & nssd & nssd \\
\hline $\mathrm{VIC} / \mathrm{NSC}$ & nssd & nssd & 0.05 & 0.01 & nssd & nssd & nssd & nssd \\
\hline \multirow[t]{3}{*}{$\mathrm{NSC} / \mathrm{NSA}$} & 0.001 & $\mathrm{nssd}$ & nssd & 0.05 & nssd & nssd & nssd & nssd \\
\hline & \multicolumn{8}{|c|}{ Two vehicles - method } \\
\hline & \multicolumn{2}{|c|}{ open } & \multicolumn{2}{|c|}{ close } & \multicolumn{2}{|c|}{ Octava } & Pan & sonic \\
\hline $\mathrm{VIC} / \mathrm{NSA}$ & $\mathrm{ns}$ & sd & & sd & & ssd & & sd \\
\hline $\mathrm{VIC} / \mathrm{NSC}$ & 0. & 01 & & 01 & & $\mathrm{ssd}$ & & $\mathrm{sd}$ \\
\hline $\mathrm{NSC} / \mathrm{NSA}$ & $\mathrm{ns}$ & sd & & sd & & 01 & & 01 \\
\hline & & & o vehic & $\mathrm{es}-\mathrm{d}$ & rection & of $\mathrm{mo}$ & tion & \\
\hline & $\mathrm{L} / \mathrm{F}-\mathrm{B}$ & $\mathrm{R} / \mathrm{B}-\mathrm{F}$ & L/B-F & $\mathrm{R} / \mathrm{F}-\mathrm{F}$ & $\mathrm{F} / \mathrm{L}-\mathrm{R}$ & B/R-1 & F/R-I & B/L-R \\
\hline VIC/NSA & 0. & 01 & & sd & & ssd & & 05 \\
\hline $\mathrm{VIC} / \mathrm{NSC}$ & 0. & 01 & & $\mathrm{sd}$ & & 05 & & $\mathrm{sd}$ \\
\hline $\mathrm{NSC} / \mathrm{NSA}$ & $\mathrm{ns}$ & $\mathrm{sd}$ & & $\mathrm{sd}$ & & 05 & & 01 \\
\hline
\end{tabular}

TABLE III

Results of the statistical analysis ANOVA of the correct answers versus the method of recording and reproduction in particular tasks; $p$ - level of significance at which the answers revealed statistically significant differences related to the method of recording and reproduction of sound events.

\begin{tabular}{l|c|c|c|c|c}
\hline \hline \multicolumn{2}{c}{ Tested groups } & \multicolumn{2}{|c|}{ Stationary sources } & \multicolumn{2}{c}{ Number of vehicles } \\
\cline { 3 - 6 } \multicolumn{2}{l|}{} & drum & rattle-box & single & two \\
\hline $\begin{array}{l}\text { Differences } \\
\text { between }\end{array}$ & VIC & 0.001 & 0.001 & 0.0000 & 0.0000 \\
\cline { 2 - 6 } & NSC & 0.01 & 0.001 & 0.034 & 0.0000 \\
\cline { 2 - 6 } methods & NSA & nssd & nssd & nssd & 0.0288
\end{tabular}

ysis results inside the groups studied versus the method of recording and reproduction for particular tasks. The level of significance at which the responses revealed differences depending on the method of recording and reproduction is given.

\subsection{Immovable sound sources}

In the first experiment, the subjects were asked to identify the direction from which the sound generated by a stationary (immovable) source had been coming. Statistical significance in the means of correct answers revealed for groups of subjects compared in the stationary sources localization task is shown in Table I. The analysis of variance of the results of the first task has shown statistically significant differences between all groups of subjects when the sound source was the drum. When 


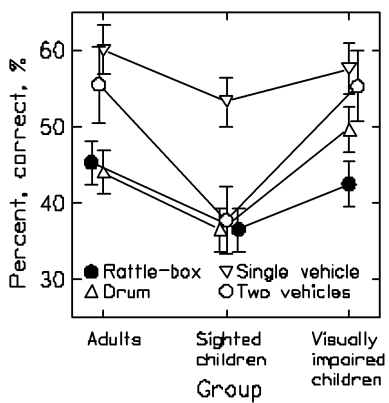

Fig. 3. The effect of the acoustic signal on the results of localization test obtained by the groups studied in particular tasks, e.g. localization of immovable source of sound, identification of direction of motion of a single vehicle, and identification of direction of motion of two simultaneously moving vehicles.

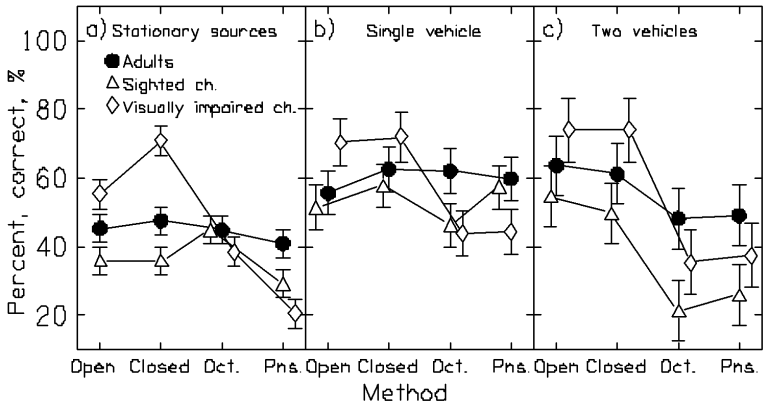

Fig. 4. The effect of the method of sound reproduction on the results of localization test obtained by the groups studied in particular types of tasks: (a) localization on an immovable source of sound, (b) identification of direction of motion of a single vehicle, (c) identification of the direction of motion of two vehicles moving simultaneously. Oct. - loudspeaker system presentation of the sounds recorded with an Octava matrix PNS loudspeaker system presentation of the sounds recorded with a Panasonic matrix.

the sound source was the rattle-box, no differences were noted between VIC and the adults (Fig. 3, Table I).

Next, the effect of the method of recording and reproduction of signals was tested, and the results are given in Fig. 4a and Table I. The most pronounced differences between the groups of subjects were noted when the sound was reproduced through closed headphones. The best results were obtained by the VIC, while the poorest by children with normal eyesight (Fig. 4a). No statistically significant differences between the groups of subjects were noted when reproducing sound recorded by the Octava matrix (Table I). When the sound recorded by the Panasonic matrix was reproduced, no differences were fund between the children with normal eyesight and the VIC.

Analysis of the influence of the method of sound recording and reproduction on the effectiveness of sound source localization inside the groups was also made, and the results are presented in Fig. 4a and Table III. In the

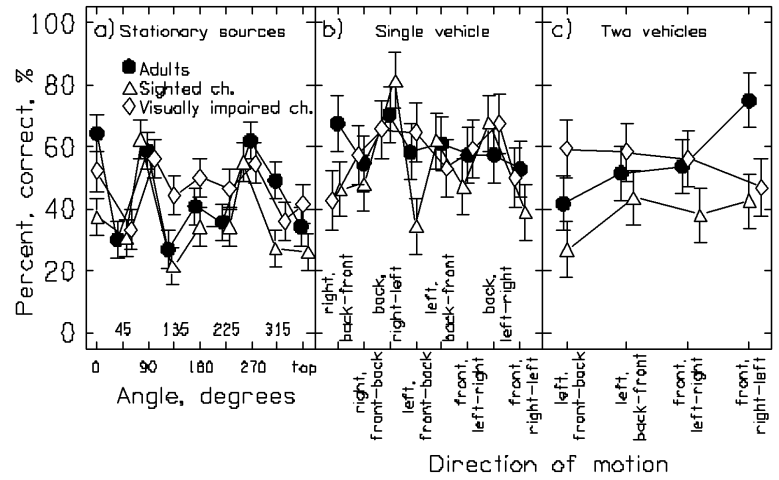

Fig. 5. The effect of direction of sound presentation on the results of localization test obtained by the groups studied in particular tasks: (a) localization on an immovable source of sound, (b) identification of direction of motion of a single vehicle, (c) identification of the direction of motion of two vehicles moving simultaneously; when the acoustic event was the movement of two vehicles, the direction of motion of one of them is specified as the other was moving in the opposite direction and on the opposite side of the recording system.

group of adults, the method of sound recording and reproduction had no effect on correct localization of the sound source as for all methods, the adults achieved the same results (Fig. 4a). For children, the poorest results were obtained for the Panasonic matrix. The VIC got the best results when the sound was reproduced through closed headphones, while the children with normal eyesight got the best results when the recording was made by the Octava matrix and reproduced by loudspeakers (Fig. 4a).

Fig. 5a and Table I present the data illustrating the effect of the direction of sound presentation on the correct localization of the sound source. For all groups the results were the same when the sound was presented at angles $45^{\circ}, 90^{\circ}$, and $270^{\circ}$. The most pronounced differences in localization of sound source were noted when the sound was produced at the angle $0^{\circ}$, directly in front of the subject, then the best results were obtained for the adults. For the sound reproduced at angles $135^{\circ}, 180^{\circ}$, and $225^{\circ}$, the best results were obtained for the VIC (Fig. 5a). Statistically significant difference between the results for adults and normal eyesight children was found only for the angles $0^{\circ}$ and $315^{\circ}$, better results were noted for the adults. For other angles the adults and normal eyesight children got the same results.

\subsection{Single vehicles}

In the second task, the subjects were asked to identify the direction of motion of a single car and the vehicle location with respect to the listener. Statistical significance in the means of correct answers revealed for the groups of subjects compared in the task with a single moving vehicle is shown in Table II. As follows from the analysis of variance, statistically significant differences were 
obtained between the children with normal eyesight and the adults (Fig. 3, Table II). No differences were noted between the normal eyesight children and VIC as well as between the adults and VIC.

In general, besides the sounds recorded by the Octava matrix, the normal eyesight children got the same result as the adults (Fig. $4 \mathrm{~b}$, Table II). The VIC got the best results when the sound was reproduced through headphones (both closed and open) and the worst when the sound was reproduced through loudspeakers and recorded by the Panasonic matrix (Fig. 4b). When the sound was recorded by the Octava matrix and reproduced by loudspeakers, the best results were noted for adults.

The most pronounced influence of the method of sound recording and reproduction was noted in the VIC. This influence was much smaller in children with normal eyesight and no influence was observed for the adults (Fig. 4b, Table III). The VIC more accurately determined the direction of motor car motion when presented the sound through headphones and not through loudspeakers. For the children with normal eyesight it can only be concluded that when the sound was recorded by the Octava method, their results were worse than otherwise.

When the influence of the direction of motion was considered, only in three cases of motion the differences were noted: when the car moved on the right hand side from back towards the front, on the left hand side from the front to the back, and behind from right to left. In the other cases no differences were found (Fig. 5b, Table II). Except for one event of the car moving on the right hand side from the back to the front, the VIC got the same results as the adults. The children with normal eyesight had the greatest problems with identification of car moving on the left hand side from the front to the back (Fig. 5b).

\subsection{Two vehicles}

In the third task, the subjects were asked to identify the directions of motion of two motor vehicles moving simultaneously. Statistical significance in the means of correct answers revealed for the groups of subjects compared in the task with two moving vehicles is shown in Table II. As follows from the analysis of variance, the VIC and the adults got the same results (Figs. 3 and $4 \mathrm{c}$, Table II). The greatest problem in identification of the directions of two sources of sound had the children with normal eyesight and their results were statistically significantly different from those obtained by the other two groups.

Analysis of correct answers given by particular groups depending on the method of sound event recording and reproduction revealed that the results obtained by the VIC were the same as those obtained by the adults, irrespective of the method (Fig. 4c, Table II). The children with normal eyesight got worse results than the VIC on sound presentation via headphones and worse results than adults on sound presentation via loudspeakers (Fig. 4c).
Analysis of the effect of the method of sound recording and presentation on correctness of answers in particular groups showed that in each group, the sound presentation via headphones gave better results than the presentation via loudspeakers (Table III, Fig. 4c).

In the task aimed at identification of direction of motion of two vehicles, all groups gave the same percentage of correct answers when one car was moving on the left hand side from the back to the front and the other car was moving on the right hand side from the front to the back (Fig 5c, Table II). The best results in the group of adults were obtained when one car was moving in front of the listener from right to left and the other behind the listener from left to right (Fig. 5c). The children with normal eyesight had the most problems with correct identification of directions of motion of two vehicles. The group of VIC got the best results when one car was moving on the left hand side from the front to the back and the other on the right hand side from the back to the right.

\section{Conclusions}

Analysis of the above-presented results has allows to draw the following conclusions.

1. For the group of adults, the method of sound recording and reproduction had little effect on the results. Only for the acoustic event of movement of two cars, the sound presentation through headphones gave better results than presentation via loudspeakers.

2. For the VIC, a statistically significant difference was noted between the results obtained upon sound presentation through headphones and loudspeakers. For sound presentation through headphones, the results were better.

3. For the group of children with normal eyesight, statistically significant differences were found between the methods of recording and presentation in each task. It is impossible to conclude which method was the best and which the worst as, depending on the task, the effect of the method was different.

4. The subjects found it easier to identify the direction of car motion than the direction of sound from immovable source and to identify the direction of motion of single cars than two simultaneously moving cars.

5. The method of binaural recording and sound presentation through headphones seems to be better for the spatial orientation training on the basis of sounds for the VIC. 


\section{Acknowledgments}

The work was partly supported by the National Science Centre (Grant no. 2012/05/B/HS6/03863).

\section{References}

[1] E. Bogusz, E. Skrodzka, E. Hojan, M. Jakubowski, A. Talukder, D. Hojan-Jezierska, Pol. J. Environm. Stud 20, 1395 (2011).

[2] E. Bogusz, H. Koprowska, E. Skrodzka, Acta Phys. Pol. A 121, A19 (2012).

[3] E. Hojan, M. Jakubowski, A. Talukder, H. Wereda A. Furmann, R. Ewertowski, E. Skrodzka, P. Perz, P. Pękala, E. Bogusz, H. Lubawy, F. Tomaszewski, B. Czechyra, M. Orczyk, G. Szymański, M. Niewiarowicz, D. Hojan-Jezierska, A. Jezierska, Acta Phys. Pol. A 121, A-5 (2012).

[4] B. C. J. Moore, An Introduction to the Psychology of Hearing, 1st ed., PWN, Warszawa 1999 (in Polish).
[5] E. Ozimek, Sound and Perception, PWN, Warszawa 2002 (in Polish).

[6] J. Blauert, in: Binaural and Spatial Hearing in Real and Virtual Environments, Eds. R.H. Gilkey and T. Anderson, Lawrence Erlbaum, USA-Mahwah NJ 1996.

[7] B.B. Bauer, J.Acoust. Soc.Am. 33, 1536 (1961).

[8] V. Pulkki, J. Audio-Eng. Soc 45, 456 (1997).

[9] J. Blauert, N. Xiang, Acoustics for Engineers, Springer, Berlin 2009.

[10] M. Gerzon, J. Audio-Eng. Soc. 21, 2 (1973).

[11] A. J. Berkhout, J. Audio-Eng. Soc. 36, 977 (1988).

[9] J. Blauert, R. Rabenstein, Proc. of 5\%th Open Seminar on Acoustics, Gliwice 2010, p. 21.

[13] K. Farrar, Wireless World 48 Oct. (1979).

[14] D.G. Malham, A. Myatt, Computer Music Journal 19, 58 (1995). 